\title{
A Study of Recrystallization and Phase Transitions in Intermetallic Titanium Aluminides by In-Situ High-Energy X-Ray Diffraction
}

\author{
K.-D. Liss ${ }^{1, a}$, A. Bartels ${ }^{2, b}$, H. Clemens ${ }^{3, c}$, S. Bystrzanowski ${ }^{2, d}, A$. Stark $^{2, e}$, \\ T. Buslaps ${ }^{4, f}$, F.-P. Schimansky ${ }^{5,9}$, R. Gerling ${ }^{5, h}$, A. Schreyer ${ }^{5, i}$ \\ ${ }^{1}$ Bragg Institute, Australian Nuclear Science and Technology Organisation, Menai NSW 2234, \\ Australia \\ ${ }^{2}$ Department of Science and Technology, Hamburg University of Technology, D 21073 Hamburg, \\ Germany \\ ${ }^{3}$ Department of Physical Metallurgy and Materials Testing, Montanuniversität, A 8700 Leoben, \\ Austria \\ ${ }^{4}$ European Synchrotron Radiation Facility, F 38043 Grenoble Cedex \\ ${ }^{5}$ GKSS Research Centre, D 21502 Geesthacht, Germany \\ aliss@kdliss.de and kdl@ansto.gov.au, ${ }^{b}$ bartels@tu-harburg.de, chelmut.clemens@unileoben.ac.at, \\ dbystrzanowski@tu-harburg.de, e'stark@tu-harburg.de, 'buslaps@esrf.fr, \\ ${ }^{9}$ Frank-Peter.Schimansky@gkss.de, 'rainer.gerling@gkss.de, 'schreyer@gkss.de
}

Keywords: titanium aluminides; phase transformation; recrystallization; grain growth; synchrotron radiation; in-situ experiment.

\begin{abstract}
High-energy synchrotron X-ray diffraction is a novel and powerful tool for bulk studies of materials. In this study, it is applied for the investigation of an intermetallic $\gamma$-TiAl based alloy. Not only the diffraction angles, but also the morphology of reflections on the Debye-Scherrer rings are evaluated in order to approach lattice parameters and grain sizes as well as crystallographic relationships. An in-situ heating cycle from room temperature to $1362{ }^{\circ} \mathrm{C}$ has been conducted starting from massively transformed $\gamma$-TiAl which exhibits high internal stresses. With increasing temperature the occurrence of strain relaxation, chemical and phase separation, domain orientations, phase transitions, recrystallization processes, and subsequent grain growth can be observed. The data obtained by high-energy synchrotron X-ray diffraction, extremely rich in information, are interpreted step by step.
\end{abstract}

Introduction. With the advent of $3^{\text {rd }}$ generation synchrotrons in the upper energy range more than a decade ago, diffraction techniques employing high-energy $\mathrm{X}$-rays have been evolved for the study of physics and materials in the field of hard condensed matter [1]. The photo absorption process becomes weak with rising energy and thus the penetration of the radiation can reach several centimeters for light to medium weighting elements and even the bulk of lead containing samples can be probed in the millimeter range. This, together with the flux available and the spatial resolution of sub-millimeter shaped beams makes X-rays in the range of $100 \mathrm{keV}-200 \mathrm{keV}$ a unique tool for structural investigations. Due to the short wavelength, diffraction angles are relatively small, typically less than $10^{\circ}$ and scattering occurs mainly in the forward-transmission direction, which can be registered simultaneously by a flat, two-dimensional detector, Figure (1). Polycrystalline samples diffract into Debye-Scherrer cones which are projected in forms of rings or ellipses onto the detector aligned with its surface normal parallel towards the incident beam to the sample. The ring diameters give direct evidence of the lattice spacing distribution in the sample. A potential eccentricity of the rings relates to residual strain of a workpiece after thermo-mechanical deformation or heat treatments. Intensity changes along the azimuthal angle relate to textures and the morphology of the rings smooth or spiky - give statistics about the grain distribution in the sample. 


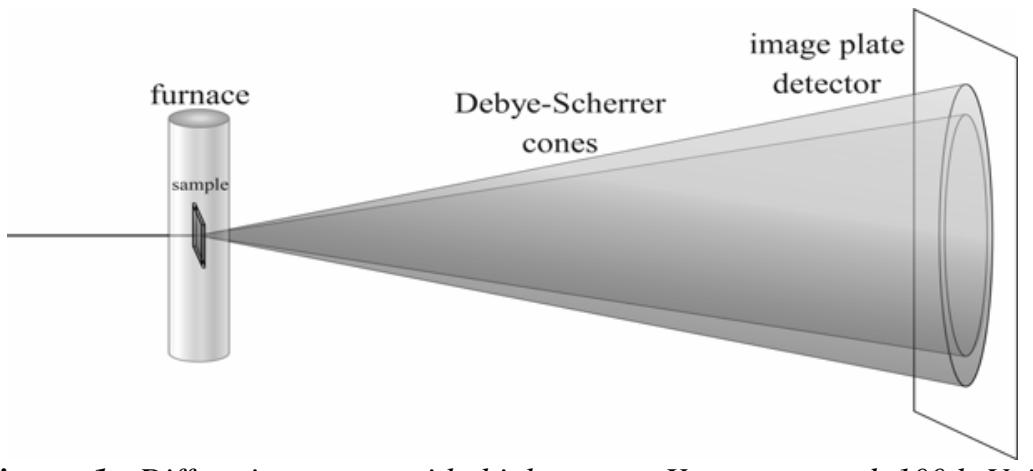

Figure 1: Diffraction setup with high-energy $X$-rays around $100 \mathrm{keV}$ in transmission for in-situ experiments. The polycrystalline sample is located in a furnace, performing powder-diffraction in the monochromatic beam which arrives from the left. Debye Scherrer rings can be recorded in the far distance.
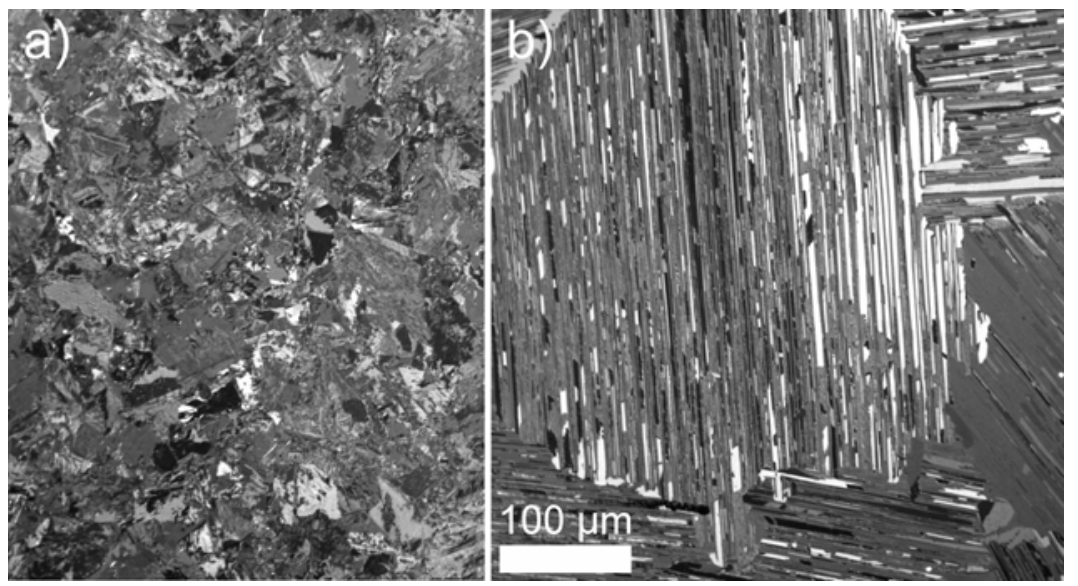

Figure 2: Microstructure of the sample before a) and after b) the heating cycle as shown in Figure (3). The massively transformed starting material recystallizes into a fully lamellar structure.

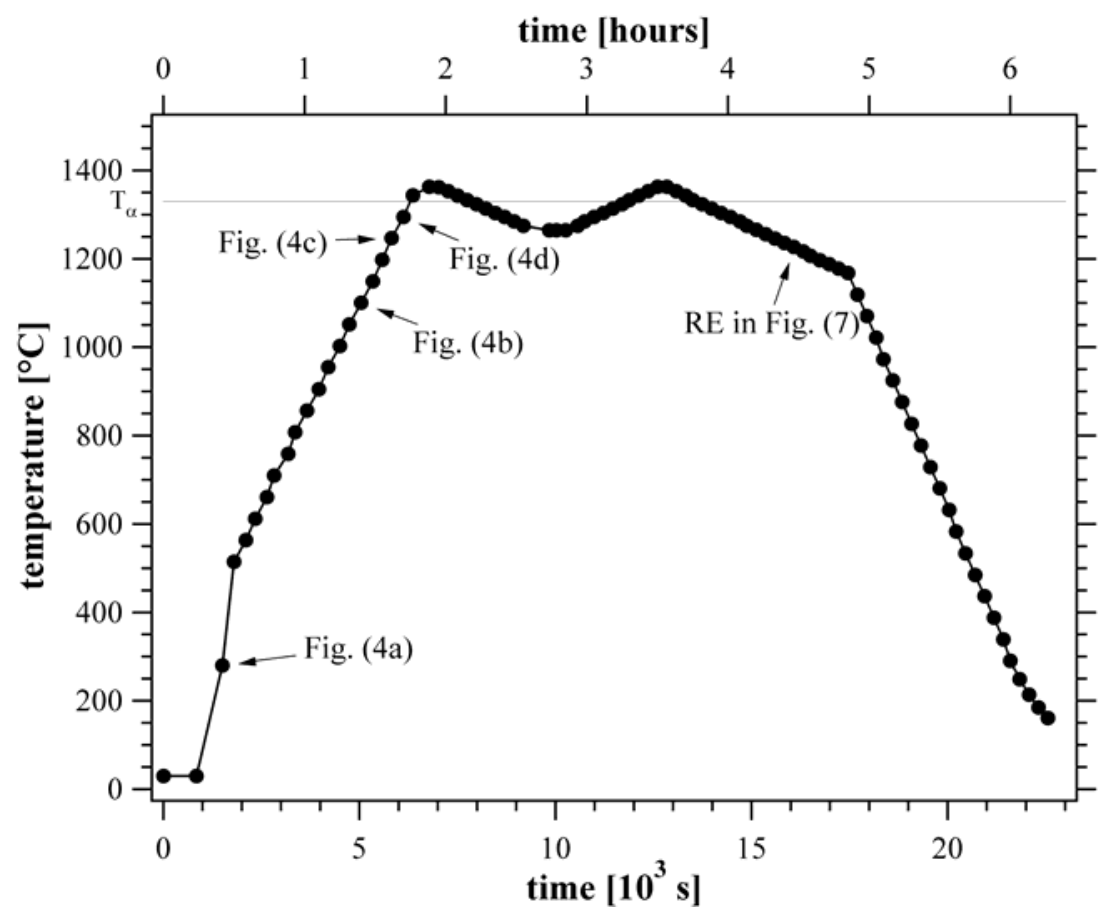

Figure 3: Temperature profile as used for the in-situ experiment with data acquisition points, full circles. The tags relate to Figs. (4) and (7). RE marks the evaluated point after re-entrance into the $\alpha+\gamma$-field from above $T_{\alpha}$.
Intermetallic $\gamma$-TiAl based alloys bear the advantage of their light weight combined with mechanical strength at very high temperature which make this material a strong candidate for heat-exposed mechanical parts such as turbine blades or turbo chargers in the aerospace and transportation industry [2]. The mechanical properties depend strongly on the prevailing microstructure, which is not only a function of composition, but largely depends on the thermo-mechanical history of the material. Therefore, the detailed understanding of the physical and chemical processes during thermo-mechanical processing or annealing are of most importance. Traditionally, samples are heated to the temperature condition of interest, then quenched, cut, polished and subsequently investigated in a thin layer at room temperature. Here we present a novel in-situ study performed by high-energy X-rays.

Experiment. The investigated sample is a niobium rich $\gamma$-TiAl based alloy of composition Ti-46Al-9Nb (atomic percent) which has been manufactured by a powder metallurgical process and then rolled into sheets [3]. Phases of interest are the $\alpha$-phase at high temperatures which transforms into a $\gamma+\alpha$ field below the $\alpha$-transus temperature $\left(\mathrm{T}_{\alpha}=1330{ }^{\circ} \mathrm{C}\right)$ while the disordered $\alpha$ orders into $\alpha_{2}$ below the eutectoid temperature $\left(\mathrm{T}_{\mathrm{e}} \sim 1170{ }^{\circ} \mathrm{C}\right)$. Both $\alpha$ and $\alpha_{2}-\mathrm{Ti}_{3} \mathrm{Al}$ are hexagonal with a distinguished superstructure in the ordered phase. For the purpose of minimal ambiguity, 
we refer always to the larger unit cell and indexation of the ordered phase for Bragg reflections and lattice parameters. The $\gamma$-TiAl phase is almost face centered cubic with a tetragonal deviation of a few percent due to alternating $\mathrm{Ti}$ and $\mathrm{Al}$ layers along the c-axis. It is known, that the $\mathrm{Nb}$ atoms substitute Ti sites, such that the system can be regarded as (Ti,Nb)Al and (Ti,Nb) ${ }_{3} \mathrm{Al}$ for the $\gamma$ - and $\alpha_{2}$-phase, respectively. The sample has been tempered at $T_{\alpha}$ for 4 minutes and then oil-quenched which results in a massively transformed $\gamma$-phase [4]. The microstructure is shown in Figure (2a) and reveals that there is no typical grain size, but a large distribution with a fractal aspect, ranging from the optical resolution limit to about $50 \mu \mathrm{m}$ diameter.

The massively transformed TiAl sample has been placed in the furnace of the diffraction setup, Figure (1), and a temperature profile according to Figure (3) run. The temperature was continuously increased above and then oscillated gently around $\mathrm{T}_{\alpha}$ before it was decreased to room temperature. The micrograph taken afterwards shows a complete recrystallized lamellar microstructure, Figure (2b). Typical colony sizes are $400 \mu \mathrm{m}$ which are made up by coherently grown lamellae of a few micrometer thick $\gamma$-laths intersected by much thinner $\alpha_{2}$-laths. The crystallographic relationship is given by the well-known Blackburn relation which states that the $\gamma-\{111\}$ plane fits well the $\alpha_{2}-\{002$ ) plane of the hexagonal phase [5]. However, 3 different domain orientations of the tetragonal c-axis are possible which results, together with a twin-operation for each domain, into 6 possible orientations of the $\gamma$-phase with respect to the $\alpha_{2}$-phase.

Diffraction patterns of the sample have been taken in-situ continuously all $275 \mathrm{~s}$, represented by the black dots in Figure (3). Extracts from some of them are represented in Figure (4). The beam size was in the order of $100 \mu \mathrm{m}$ and the X-ray energy calibrated to $94.4 \mathrm{keV}$. The sections of the rings are indexed and show both phases. The Debye-Scherrer rings are not continuous as one would expect them to be in a good powder average but show a series of more or less continuously distributed spots of different intensities. This is due to the relatively small beam size with respect to the grain size of the material. Each sharp spot is a single crystal reflection of a particular grain and the azimuthal distribution around it is its mosaic spread.

Results and Discussion. At $280^{\circ} \mathrm{C}$, Figure (4a), the pattern is exclusively composed by a contribution of the $\gamma$-phase and looks very blurred. Particularly, the $\gamma-002$ and $\gamma$-200 reflections should feature a double ring due to the slight difference in lattice spacing along the $\mathrm{c}$ and the a axis.

(a)

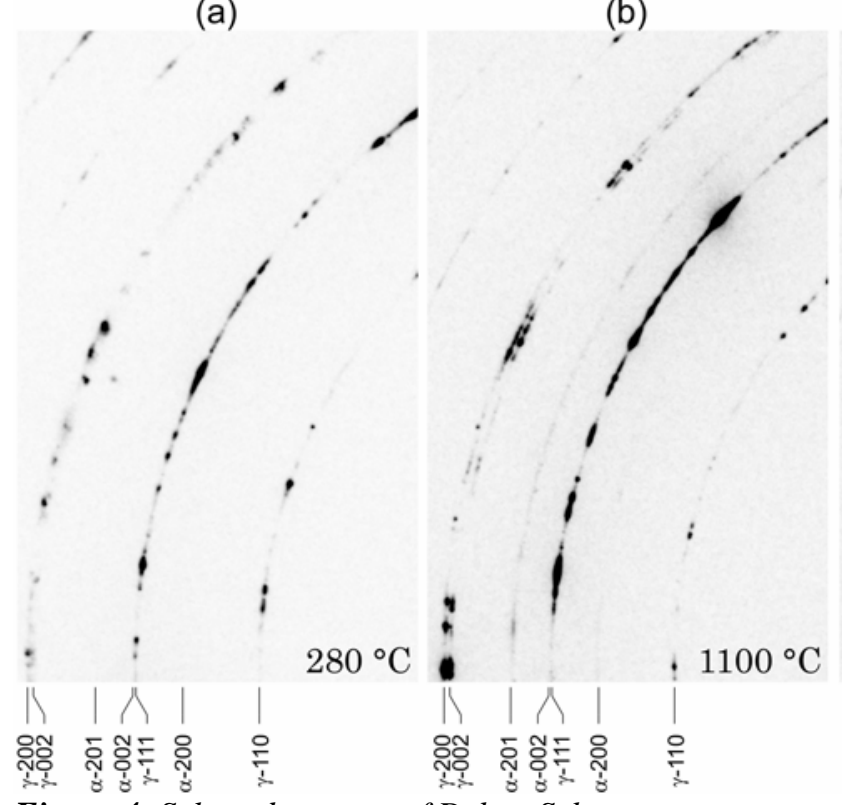

(c)

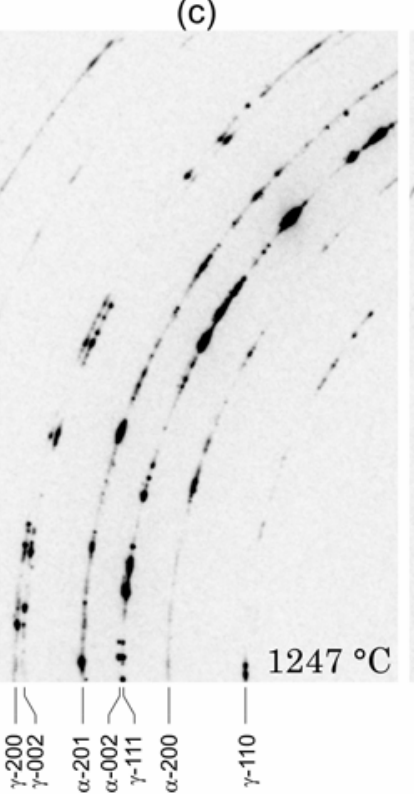

(d)

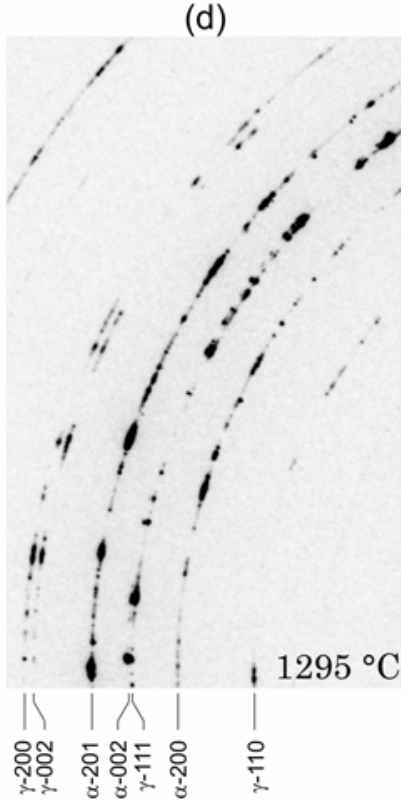

Figure 4: Selected extracts of Debye-Scherrer patterns recorded in-situ at the given temperatures upon heating. The transformation from massively transformed $\gamma$-phase (a) through the $\gamma+\alpha$-field $(b, c, d)$ close to $T_{\alpha}$ is clearly seen and shows many features along the rings. 
This splitting is washed out, not to a single sharp ring as in a cubic structure, but to a broad distribution between the reciprocal $\mathrm{c}$ and a values. It attests to huge internal stresses and microstructural disorder stemming from the nucleation and growth process of the massively transformed material when $\gamma$-domains grew spontaneously all over a parent $\alpha$-grain, each with the c-axis oriented arbitrary in one of the preferred directions. After the whole material had transformed, domains touched each other and the misfit stores a large amount of mechanical energy. Since disorder is high, domains are small such that the stress cannot relax. The pattern corresponds well to the fractal appearance of the micrograph in Figure (2a).

First, the micro-stresses relax with increasing temperature and the expected double-ring feature of the $\gamma-002$ and $\gamma$-200 reflexes appears which we attribute to the re-orientation of smaller domains in favor of a larger. This recovery process starts above $700{ }^{\circ} \mathrm{C}$ and both rings are already well distinguished in Figure (4b). Second, re-arrangement of the spots on the Debye-Scherrer rings occurs and, in particular, weaker reflections disappear proofing for grain growth and related change in the phase ratio, which is seen particularly in Figure (4c,d), where a major amount of $\alpha$-phase is present. From $1100{ }^{\circ} \mathrm{C}$ to $1295{ }^{\circ} \mathrm{C}$, the morphology of the ring-patterns changes completely and gives evidence for an entirely re-arranged microstructure, still entangled by the coexistence of two phases. It is worth to note, that reflections on the $\gamma-002, \gamma$-200 rings mostly appear in pairs or agglomerates, sitting either face-to-face on both rings or sometimes shifted by a small azimuth angle $\Delta v$. Such a situation is magnified in Figure (5a) and relate to a small-angle domain boundary as sketched in

a)

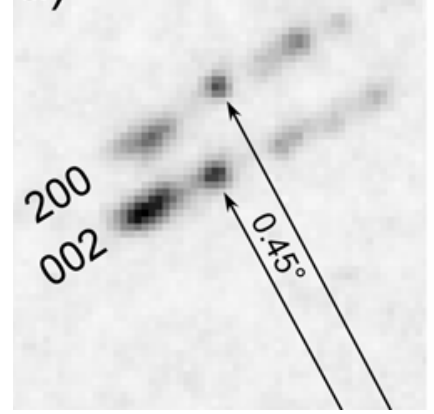

b)

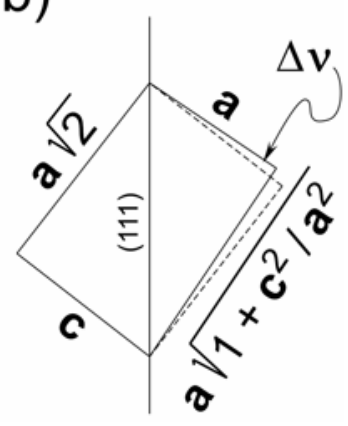

Figure 5: Correlated reflections on the $\gamma$-200 / $\gamma$-002 ring pair (a) which stem from a domain boundary (b).

Figure (5b): Neighboring domains can favorably grow together on a common $\{111\}$ plane with the a and c-axes interchanged on each side of the domain boundary. The angle can be calculated by simple geometric considerations and with a ratio of $\mathrm{c} / \mathrm{a}=1.011$ obtained from the ring diameters, results in a shift of $\Delta v=0.45^{\circ}$, which fully explains the observed maximal angular shift. Smaller angles can be observed due to a geometrical projection of the shift along the beam direction. String-of-pearl like reflection patterns can be recognized and are explained by successive domain-angle shifts. The detailed look to the $\alpha-002 \gamma-111$ pair gives similar evidence of face-to-face reflections which relate directly to the Blackburn relation between the two phases as discussed above. Accordingly, the ring triplet $\alpha-220, \gamma-202, \gamma-220$ is shown magnified in Figure (6) and demonstrates coherent relations in a second orientation between the two phases and the domains in the $\gamma$-phase which are perpendicular to the hexagonal base plane and the common $\{111\}$ planes, respectively. The longitudinal variation of the scattering reciprocal lattice vectors, i. e. the radii of the Debye-Scherrer rings in Figure (6), can be evaluated by azimuthal integration over a representative sector of the rings which leads to a classical powder diffraction pattern. The evolution of the position of individual reflection lines can be evaluated as a function of temperature and is plotted in Figure (7a). The reciprocal lattice values of the $\gamma$-111 reflection shrink with increasing temperature which is due to thermal expansion. Only the last two data points below $\mathrm{T}_{\alpha}$ deviate systematically, since the contribution of the closely nearby $\alpha-002$ reflection becomes strong and
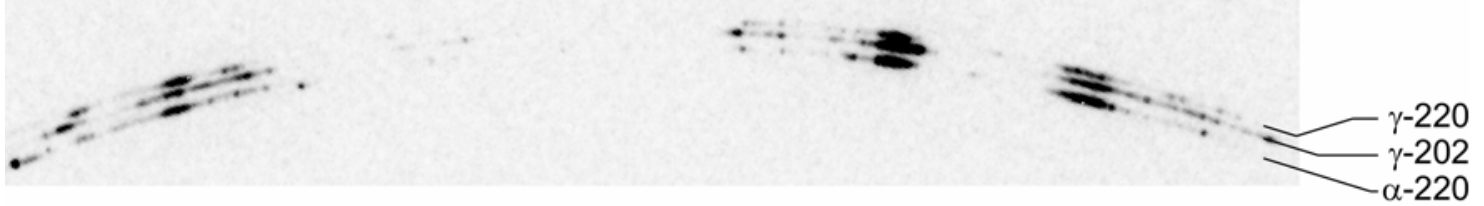

Figure 6: Correlated reflections on the $\gamma-220, \gamma-202, \alpha-220$ ring triplet proofing the Blackburn relation between the two phases. 
falsifies the result. The gray line is a fit to the data (excluding these 2 points) and delivers

$$
\Delta \mathrm{G} / \mathrm{G}(\mathrm{T})=-8.8909 \cdot 10^{-6} \mathrm{~T} /{ }^{\circ} \mathrm{C}-2.6057 \cdot 10^{-9}\left(\mathrm{~T} /{ }^{\circ} \mathrm{C}\right)^{2}
$$

for the expansion coefficient which is in excellent agreement with the literature and even extends the temperature range up to $T_{\alpha}[6,7]$. The relative variation of the $\gamma-200$ reflection follows well this behavior. Since the c-axis has a slightly smaller thermal expansion than the a-axis [6, 7], the $\gamma-200$ values tend to lie little below the $\gamma-111$ values. Only the data point just below $\mathrm{T}_{\alpha}$ deviates from this behavior upon heating. The scattering vectors for the $\gamma-002$ reflection have been normalized to the room temperature value of $\gamma-200$ and show a room temperature offset of 1 -c/a $=-4.3 \cdot 10^{-3}$ due to the tetragonal splitting. It evolves similar to the described thermal expansion until $\sim 930{ }^{\circ} \mathrm{C}$. Above this temperature, the curve continuously deviates from the mean behavior until $\mathrm{T}_{\alpha}$ with another kink at the eutectoid temperature of $\sim 1170{ }^{\circ} \mathrm{C}$. The related c/a ratio is derived directly and plotted in Figure (7b). Obviously, the $\gamma$-phase and the related reflections disappears at $\mathrm{T}_{\alpha}$. After slow cooling back into the $\alpha+\gamma$-field, $\gamma$-reflections reappear with the positions marked with an arrow in Figure (7). They lie much further apart and off the curves obtained upon heating.

We interpret the data as following: At the beginning of the experiment the Ti-46Al-9Nb sample possessed a massively transformed microstructure. A massive transformation is achieved by quenching from the $\alpha$-phase field, where the chemical elements are distributed homogeneously. During quenching $\alpha$ transforms to massive $\gamma$. Due to the high cooling rate long-range diffusion and thus phase separation is suppressed. Therefore, the massively transformed

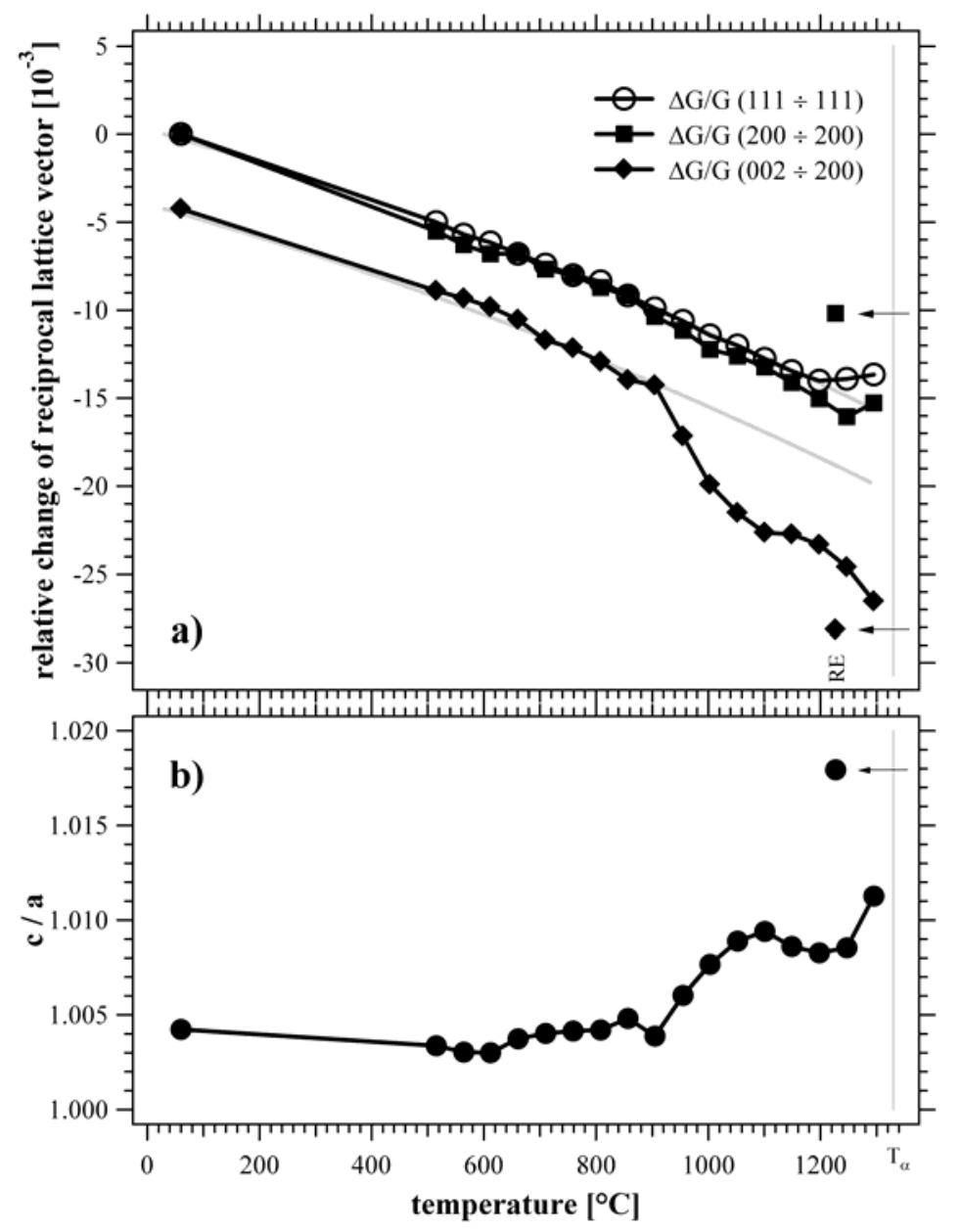

Figure 7: Temperature dependence of (a) reciprocal lattice vectors and (b) c/a ratio of the $\gamma$-phase. The arrows mark the data points RE, see Figure (3), after re-entering the $\gamma+\alpha$-phase field from above $T_{\alpha}$. $\gamma$-phase is far from stoichiometry which relates in atomic disorder and leads, in addition to the internal stress blurring, to a small splitting of the c/a ratio only. Upon ramp heating, diffusion becomes significant above $\sim 930{ }^{\circ} \mathrm{C}$ and $(\mathrm{Ti}, \mathrm{Nb})$ atoms segregate into regions where they form the Al-poor $\alpha_{2} / \alpha$-phase. The remaining material approaches continuously the stoichiometry of the $\gamma$-phase, decreasing the 'cubic' disorder part and increasing the c/a ratio. The kink around the eutectic temperature is due to latent heat which is needed for the order-disorder $\left(\alpha_{2}-\alpha\right)$ phase transition and taken off the diffusion process. Diffusion continues until we lack the $\gamma$-phase. Just below $\mathrm{T}_{\alpha}$, rearrangements are rapid and the c/a ratio increases dramatically. Inversely, upon gentle cooling below $T_{\alpha}$, this segregation process has enough time to split further to the observed values. Like in a Differential Scanning Calorimetry (DSC) experiment, none of the phases is in thermodynamical equilibrium which results in an unexpected behavior not only in the c/a 
ratio but also in the lattice parameters mismatch between the $\alpha$ - and the $\gamma$-phase. From Figure (6) we reckon $G(\alpha-220)<G(\gamma-202)<G(\gamma-220)$ which may explain the observed asymmetry in the thermal expansion of the two $\gamma$-reflection. Altogether, the lattice has to transform into smaller reciprocal lattice values while the segregation process would tend to increase the $\gamma$-200 and decrease the $\gamma-002$ component. These counter-acting tendencies for $\gamma$-200 lock in the value to the normal thermal expansion while the splitting is solely absorbed by the $\gamma-002$ component. Again, just below $\mathrm{T}_{\alpha}$, diffusion becomes so fast, that both values deviate off the normal thermal expansion. The re-entrant values match excellently a $\Delta \mathrm{G}(\gamma-200): \Delta \mathrm{G}(\gamma-002)=1: 2$ ratio which would be expected upon conservation of the volume of the unit cell, normalized to normal thermal expansion.

Conclusion and Outlook. We have studied the behavior of a previously massively transformed Ti-46Al-9Nb sample in-situ with high-energy X-rays. Not only classical powder diffraction patterns, revolving the chemical and thermal expansion behavior have been evaluated, but also the morphology of the rings was regarded for the first time. This gives information about three-dimensional correlations across domain and phase boundaries. The data set is extremely rich in information and only a few aspects could be exposed here. Further comparisons with ex-situ data are consistent and will be published elsewhere [8]. The recrystallization process at temperatures close to $\mathrm{T}_{\alpha}$ is very rapid. Higher time-resolutions well below a second for the detector will be necessary in future to follow continuously this evolution. At the end, we plan to compare the diffraction data directly with in-situ laser scanning confocal microscopy, such as practiced on other materials [9].

\section{References}

[1] K.-D. Liss, A. Bartels, A. Schreyer, H. Clemens: "High energy X-rays: A tool for advanced bulk investigations in materials science and physics", Textures and Microstructures 35-3/4 p. 219-252 (2003)

[2] H. Clemens, H. Kestler: "Processing and applications of intermetallic $\gamma$-TiAl-based alloys", Advanced Engineering Materials 2-9 p. 551-570 (2000)

[3] R. Gerling, A. Bartels, H. Clemens, H. Kestler, F. P. Schimansky: "Structural Characterization and Tensile Properties of a High Niobium Containing Gamma TiAl Sheet Obtained by Powder Metallurgical Processing", Intermetallics 12 p. 275-280 (2004)

[4] A. Bartels, S. Bystrzanowski, H. Chladil, H. Leitner, H. Clemens, R. Gerling, F.-P. Schimansky: "Massive Transformation in High Niobium Containing TiAl Alloys", Materials Research Symposium Proceedings 842 p. 5.48.1-6 (2005)

[5] M. J. Blackburn,. in "The Science, Technology and Application of Titanium Alloys", Plenum Press (1970) p. 633

[6] Y. He, R. B. Schwarz, T. Darling, M. Hundley, S. H. Whang, Z. M. Wang: "Elastic Constants and Thermal Expansion of Single Crystal Gamma-TiAl from 300 to 750 K", Materials Science and Engineering A - Structural Materials Properties Microstructure and Processing 240 p. 157-163 (1997)

[7] M. Zupan, K. J. Hemker: "High Temperature Microsample Tensile Testing of Gamma-TiAl", Materials Science and Engineering A - Structural Materials Properties Microstructure and Processing 319 p. 810-814 (2001)

[8] K.-D. Liss, A. Bartels, H. Clemens, S. Bystrzanowski, A. Stark, T. Buslaps, F.-P. Schimansky, R. Gerling, A. Schreyer: "Recrystallization and phase transitions in a $\gamma$-TiAl based alloy as observed by ex- and in-situ high-energy X-ray diffraction", Acta Materialia submitted (2005)

[9] D. Phelan, R. Dippenaar: "Widmannstätten Ferrite Plate Formation in Low-Carbon Steels", Metallurgical and Materials Transactions A 35A p. 3701-3706 (2004) 